Category: Simulation and Skills competency

\title{
A view of students learning experience \& technical challenges in biology online practical sessions.
}

\author{
Jayaseelan, S.E. \\ International Medical University, Malaysia
}

\section{Background:}

Since Covid-19 pandemic began last year, the education field faced its own challenges, pushing both educators and learners to online, literally overnight. Unlike theoretical knowledge, there's a challenge to impart practical skills via online, as it's something that can only be acquired via face-to-face (FTF) teaching. In FIS, we commenced online practical's using YouTube video references and slowly introduced 360 cameras, as FTF sessions resumed. As cases started increasing again in Oct 2020 and lessons reverted online, our latest intake students (FS320) missed the FTF practical's. We then introduced live-streaming and pre-recorded videos for them. During the live streaming, they faced many lag issues and they preferred the pre-recorded videos instead. This study was done to gauge students' perspective on their online practical experience and to identify gaps to devise improvements for future intakes. As online sessions demand reliable internet connectivity and gadgets, we wanted to find out the reason behind their technical issues, to plan better delivery methods.

\section{Methods:}

This was a qualitative study, where 20 students were randomly selected to complete a short survey, followed by focus group interviews in groups of 5 , to discuss a few key points.

\section{Results:}

From the study, we found that majority students used high-speed home internet and didn't face major connectivity issues. Instead, it was noted that the resolution and video quality of the live streaming were poor, and the lags made it worse. They preferred pre-recorded videos, which can be watched anytime. Students also suggested we provide online simulations, so they get familiarized with the practical via interactive learning.

\section{Conclusion:}

The lags were not due to students' internet coverage or geographical reasons, but poor live-streaming connectivity via Teams. It's recommended that we continue with pre-recorded videos and get IT department's intervention for upcoming live-streaming attempts, to provide students with wholesome online practical learning.

Keywords: Simulation, practical 